\title{
Learning from Discovering: An unsupervised approach to Geographical Knowledge Discovery using street level and street network images
}

\author{
Stephen Law \\ The Alan Turing Institute \& \\ University College London \\ London, UK
}

\author{
Mateo Neira \\ The Alan Turing Institute \& \\ University College London \\ London, UK
}

\begin{abstract}
Recent researches have shown the increasing use of machine learning methods in geography and urban analytics, primarily to extract features and patterns from spatial and temporal data. Research, integrating geographical processes in machine learning models and, leveraging on geographical information to better interpret these methods had been sparse. This research contributes to the ladder, where we show how latent variables learned from unsupervised learning methods can be used for geographic knowledge discovery. In particular, we propose a simple and novel approach called Convolutional-PCA (ConvPCA) which are applied on both street level and street network images in finding a set of uncorrelated visual latent responses. The approach allows for meaningful explanations using a combination of, geographical and generative visualizations to explore the latent space, and to show how the learned embeddings can be used to predict urban characteristics such as street-level enclosures and street network density.
\end{abstract}

\section{KEYWORDS}

urban analytics, unsupervised learning, convolutional neural networks, knowledge discovery, computer vision

\section{INTRODUCTION}

According to [21], Geographic knowledge discovery (GKD) is the process of using computational methods and visualization to explore spatial databases to discover useful geographic knowledge. Despite the increasing availability of spatio-temporal data and the subsequent use of machine learning to retrieve geographical knowledge, the majority of the research have mostly focused on learning a specific objective. For example, on the use of convolutional neural networks to make inferences for an output such as perceived safety [23], house price [18] and scenicness [27]. These researches require effort on both collecting the data and on learning a specific objective.

This study contributes to these research questions and proposes a model called Convolutional-PCA (ConvPCA) that summarises urban imagery into a set of lower dimensional latent responses. We apply this method to Google StreetView images[10] and Open Streets Maps (OSM) street network images [24]. In the experiments, we first map and visualise the extremes of the responses to explore spatial patterns and interpret the data geographically and visually. We then study the latent responses by associating it to different geographical datasets such as street enclosure for the StreetView data and network density for the street network data. The research finds that the visual response from the ConvPCA model has interpretable meaning with associations to geographical labelled data. From a machine learning perspective, we gain new knowledge about these latent responses which contributes to the recent efforts in linking the two disciplines [17] [25].

\section{RELATED WORKS}

\subsection{StreetViews}

Street-level images have been used extensively in smart transportation. Specifically on the deployment of autonomous vehicles where convolutional neural networks $(C N N)$ had been applied for urban scene understanding [26]. More recently, we have also seen the use of Generative Adversarial Networks (GAN) to synthetically create street scenes that could be used to train self-driving vehicles [31]. Despite its popularity in transportation research, there had been limited effort on using street-level imagery to recover geographical knowledge. One such example is StreetScore where [23] collected human perception data from street images through a crowd-sourced survey (Place Pulse 2.0) which are then used to predict the perceived safety of a place [8]. Another example is the work of Gebru et al. [9] whom extracted features such as car types from Google StreetView images to predict the income, race, education, and voting patterns for cities in the States. We have also seen the use of urban images [27] to predict scenicness ratings which were found to affect urban wellbeing. These recent works rely on extracting an interpretable medium level feature from street-level images. In contrast to these works, Law et al [18] extracted visual responses of StreetView images directly from house price. A distinguishing difference here is that the method did not extract an interpretable medium-level variable from an image but rather a general visual response that corresponds directly to house price. Our research extends from this work but rather than learning a visual response that estimates house price, we propose a method to learn a set of generic visual summaries using an unsupervised learning approach. These summaries can be interrogated, perturbed and studied for knowledge discovery.

\subsection{Street Networks}

In the case of street networks, there has been a long-standing effort to analyse and to understand them from a quantitative perspective and to generate models that are able to reproduce their empirical features. Previous works have largely been based on complexity theory and network science perspective $[5,19,29]$. This includes analyzing the spatial configuration of urban street networks [13] and 
analyzing urban systems from an information theoretic perspective [2].

More recently, there has been an increased interest in applying machine learning methods to extract useful information from the vast amount of data now openly available from sources such as OSM. Examples of such work have used neural networks to classify street network patterns of different cities, where two different methods have been used. The first uses a $C A E$ to create dense urban vectors that are used to cluster similar urban morphologies using a selforganinzing map [22]. The second approach uses a Variational Auto Encoder $V A E$ to measure similarity across different networks [15].

Generative models have also been used to generate synthetic street networks. Variational Autoencoders trained on street network images have been use by sampling from the latent space $z$ [15], however the resolution of these are low, and fail to capture fine grain detail of local streets. Generative Adversarial Networks such as StreetGAN [12] has also been proposed to generate a multitude of arbitrary sized street networks that faithfully reproduce the style of the original datasets.

Current limitations in the use of VAE, CAE, and GANs on street networks lie in the interpretability of the latent space and it's relationship to geometrical and topological properties used in established network measures. Our research contributes to these by developing a methodology to interpret the low-dimensional embedding learnt by a convolution auto-encoder. This allows for greater control on the generative model, as well as providing some initial results as to the relationship between the embedding and established network measures.

\section{METHODS AND MATERIALS}

\subsection{Convolutional-PCA}

We propose here the Convolutional-PCA (ConvPCA), which combines a type of Convolutional Neural Network called the Convolutional Auto Encoder $(C A E)$ with a linear PCA $\left(P C A_{\text {lin }}\right)$ to retrieve a set of visual responses that summarises a StreetView image or a street networks image. We first describe the $C A E$ followed by the $P C A_{\text {lin }}$. Deep Convolutional Autoencoder $C A E$ are unsupervised methods that uses convolutional neural network $(C N N)$ to extract image features $[3,11,20]$. Deep CAE consists of two set of layers, an encoder $f_{w}(\cdot)$ and a decoder $g_{u}(\cdot)$

$$
\begin{aligned}
& f_{w}(x)=\sigma(x \star W) \equiv z \\
& g_{u}(z)=\sigma(z \star U)
\end{aligned}
$$

where $x$ is the input vector, $z$ is the latent features, $\star$ is the convolution operator that extract image features and $\sigma$ is a typical activation function such as ReLU to model nonlinearity in the neural network. These convolutional layers can be stacked sequentially where the encoding layer reduces the dimension to a latent variable $z$ while the decoding layer increases the dimension back to image space. The sequential architecture can be seen in figure 1 .

Following [20], the parameters of the encoder $z=F_{w}(x)$ and the decoder $x^{\prime}=G_{u}$ are updated by minimising the reconstruction errors between $x$ and $x^{\prime}$.

$$
L_{r}\left(x_{i}, x_{i}^{\prime}\right)=\frac{1}{n} \sum\left|x_{i}-G_{u}\left(F_{w}\left(x_{i}\right)\right)\right|^{2}
$$

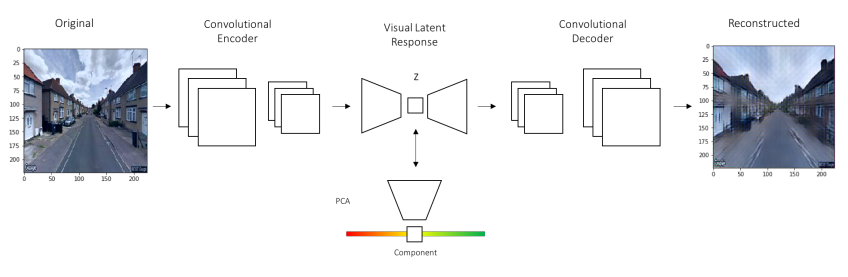

Figure 1: Architecture of ConvPCA, which combines a Convolutional AutoEncoder $(C A E)$ with a linear PCA $\left(P C A_{\text {lin }}\right)$ to retrieve a set of uncorrelated visual latent responses that summarises street-level and street-network image data.

In our research, we further compress the latent visual features by applying a linear principal component analysis $P C A_{\text {lin }}$ which summarises the visual feature $z$ into a set of linearly uncorrelated variables $v$. To compute PCA, we first standardise $z$ and compute the Eigenvectors and Eigenvalues of the feature covariance matrix $P$. We then take the Eigenvectors to calculate the full principal component decomposition of $z$, given by $V=X W$, where $W$ is the eigenvector matrix. $V$ can be re-projected back on to the original latent space produced by the encoder before passing in to the decoder to reconstruct the images. This process allows us to:

- Retrieve a set of uncorrelated visual features that can be mapped and interpreted geographically.

- Make changes to individual features to test their response in the generator.

- Relate learnt visual features to geographical labelled data.

\subsection{Materials}

We collected two datasets. The first dataset is street images taken from the Google StreetView API [10 $]^{1}$. Differ from [18], two buildingfacing images were collected for each street in the Greater London Area. To collect the dataset, we constructed a line-graph from the street network of London (OS Meridian line2 dataset [30]). We then take the geographic median and the azimuth of the street edge to give both the location and the bearing when collecting each image. We collected a total of 110, 493 street images in London. For more details in the data collection method please see [18]. Figure 3 illustrates typical images from the dataset.

The second dataset is the street network dataset taken from Open Streets Maps [24], we query all the cities and towns for a total of 107,973 . For each city and town we download the street network within a $1.5 \mathrm{~km} \times 1.5 \mathrm{Km}$ box at the centroid of each place using osmnx [4], Figure 4. For each $1.5 \mathrm{~km} \times 1.5 \mathrm{~km}$ grid we retrieve a graph $G=(V, E)$ where each vertex $v$ corresponds to a street intersection and $e$ edge corresponds to a street segment. For each $G$ we rasterise into a $256 \times 256$ pixel image Figure 5, we also calculate basic network features [6] that are latter used to test the learnt features of the images.

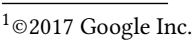




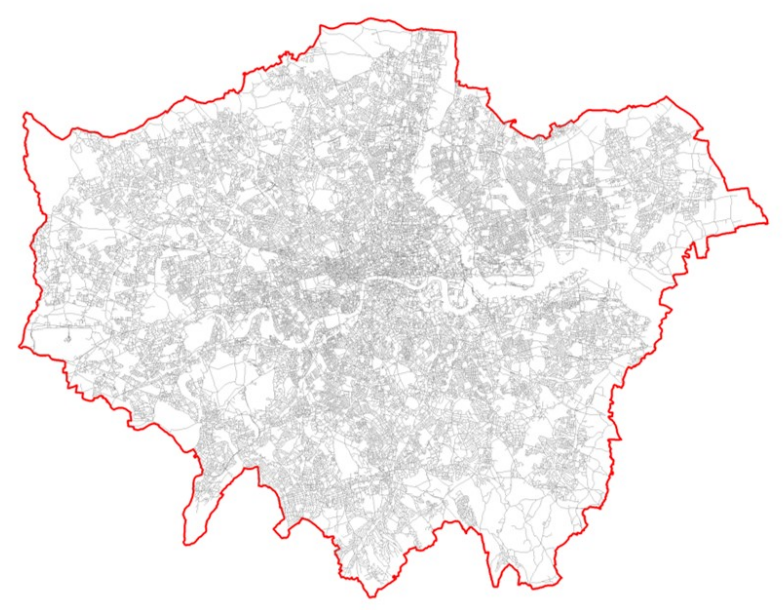

Figure 2: Greater London case study

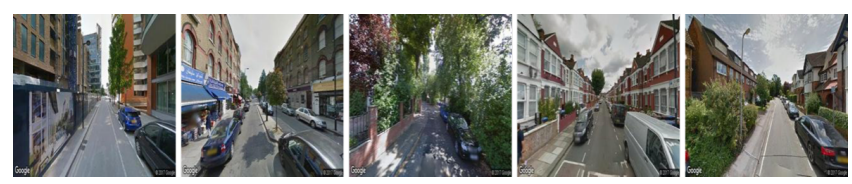

Figure 3: Samples of street level images from Google StreetView. ๑2017 Google Inc.

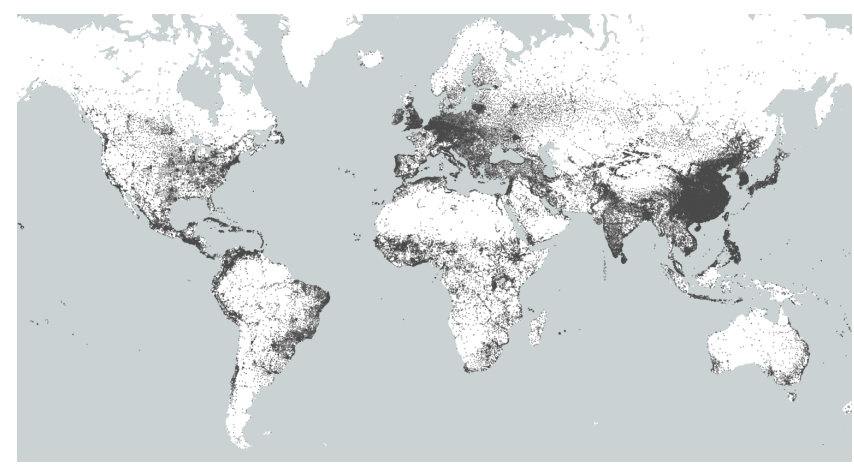

Figure 4: Centroid of cities 107,973 cities and towns used for training and validation

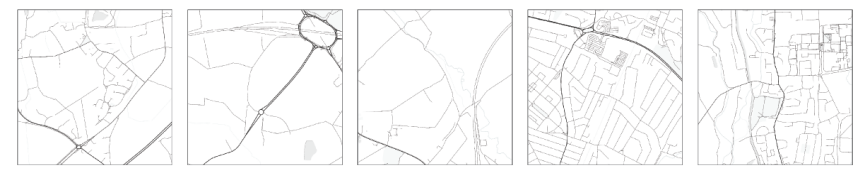

Figure 5: sample of rasterized street network data.

\section{EXPERIMENTAL RESULTS}

In order to discover new knowledge and interpretations from these visual responses, we will visualise these latent response followed by a prediction model for both types of data.

\section{1 streetview images}

4.1.1 Visualisation experiments. The ConvPCA first learns a mapping from a three channel street level images $(224 \times 224 \times 3)$ down to a lower dimensional embedding (4,096 dimensions) using an autoencoder. The lower dimension embedding was then further summarised into a set of uncorrelated responses using $P C A_{\text {lin }}$. For the autoencoder, we adopted a VGG16 [28] as the basis of the architecture where we keep the kernel size and filter numbers constant across both the encoder and decoder.
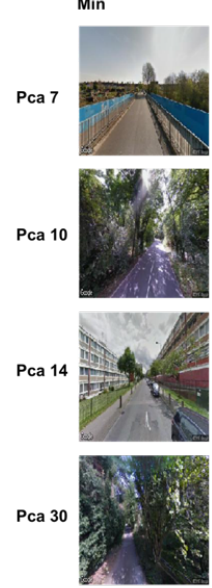
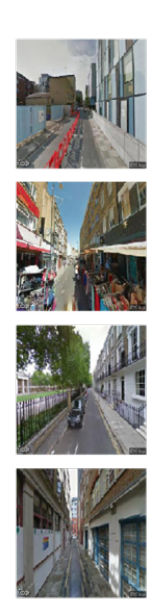
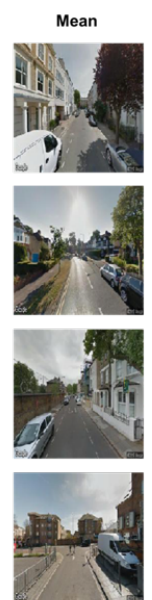
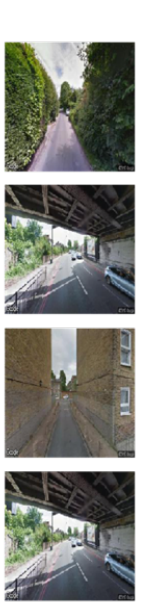
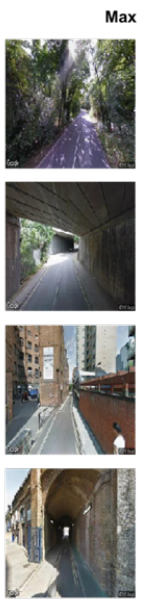

Figure 6: Visualising a sample of London Google StreetView components.

To show the results, we visualise the first two components in figure 7. The images plotted above the map show the two extremes of the visual response. We interpret the first visual response $v_{1}$ as a proxy for urban richness while we interpret the second visual response $v_{2}$ as a measure for urban density.

We then visualise the extreme values of four other components for further interpretation. In this case, component $7^{\text {th }}$ has blank facade in one of the extremes and natural scenaries in the other. The $10^{\text {th }}$ and the $30^{\text {th }}$ component shows a tunnel space in one extreme and a mixture of urban scenaries in the other. While the $14^{\text {th }}$ component has buildings in one extreme and blank facades in the other extremes. Later components shows less patterns. Further work in visual explanation is needed to better understand these visual summaries. 


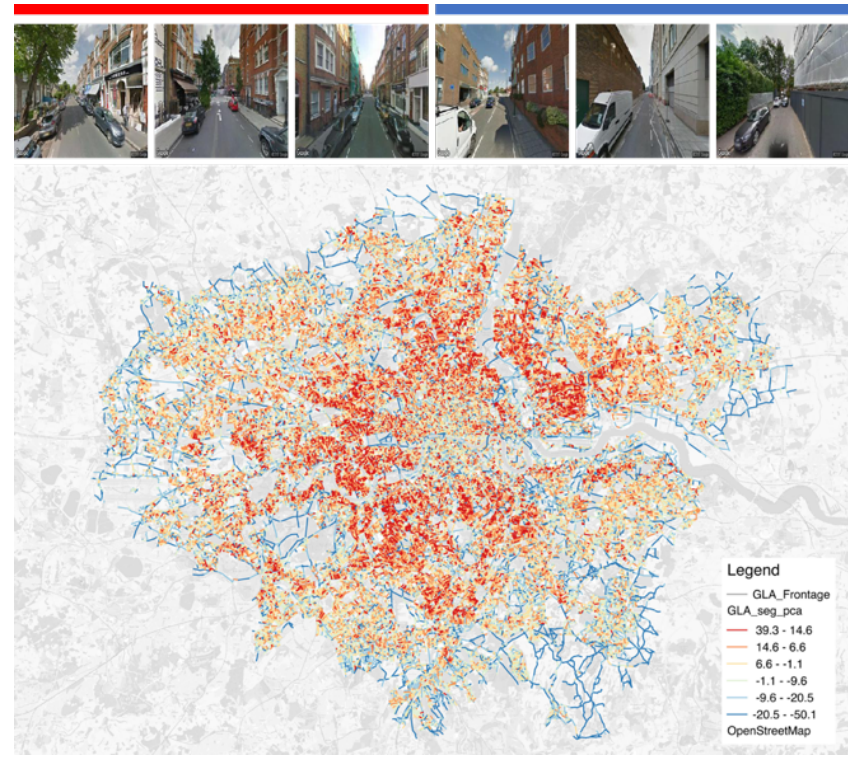

Figure 7: London StreetView Urban Richness. We interpret this component as a measure of urban richness, where red denotes higher urban richness and blue denotes lower urban richness.
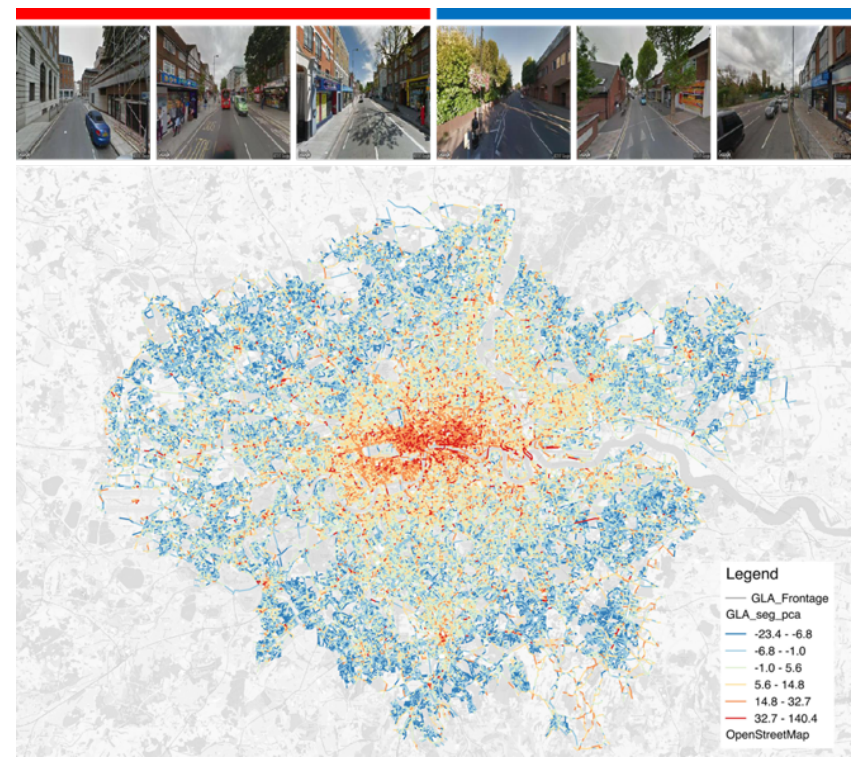

Figure 8: London StreetView Urban Density. We interpret this component as a measure of urban density or building intensity where red denotes higher density and blue denotes lower density.

Furthermore, we visualised one of the StreetView images and perturbed its first principal component while holding all the other component values constant. The plot shows when we perturbed the images towards one of the axis, building details increase, and when we perturbed the images to the other axis, the trees start filling the view. These results show geographical and generative visualisations are useful approaches to interrogate and to discover the meaning of these visual latent responses.
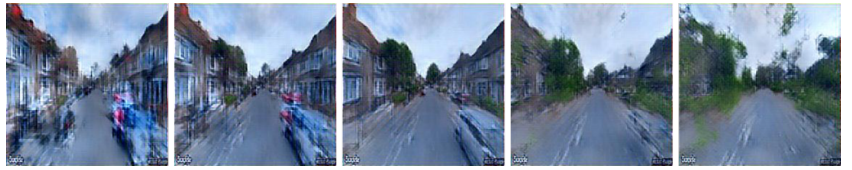

Figure 9: Visual response perturbations of a London StreetView image. By perturbing the first principal component of a typical StreetView, we show greater building details in one of the extremes and greater urban greenery in the other extreme.

4.1.2 Prediction experiment. In order to interpret these visual response and demonstrate its usefulness, we regress a set of visual responses to predict a generic urban characteristic such as street enclosure. Street enclosure here is defined as the average height of the building of a street divided by the average width between the buildings of the same street illustrated in fig 10 . This measures had been computed using GIS with Ordnance Survey data [30]. For each street in London, we split the dataset randomly into a train $(70 \%)$, validation $(15 \%)$ and test set $(15 \%)$. We then train a multilayer perceptron $F(\cdot)$ to predict normalised street enclosure from the visual response $V$ as inputs, parameterized by a set of weights $W_{v}$. The multi-layer perceptron here is defined as a fully connected neural network with two hidden layers. The first fully connected layer has 64 hidden nodes, while the second layer has 32 hidden nodes. A dropout layer was included for better generalisation. To test the importance of the visual response with respect to the model accuracy, we constructed four different models based on the number of components $[4,8,16,32]$ using the same architecture for all models. We train the model to minimize the mean squared error on a training set, using the ADAM [16] optimizer with the initial learning rate set at 0.001 . We then report the mean squared error (MSE) and the coefficient of determination $R^{2}$ between the model prediction and the street enclosure for the test-set. All the experiments are conducted with the Keras library [7] using a Tensorflow [1] back-end. 


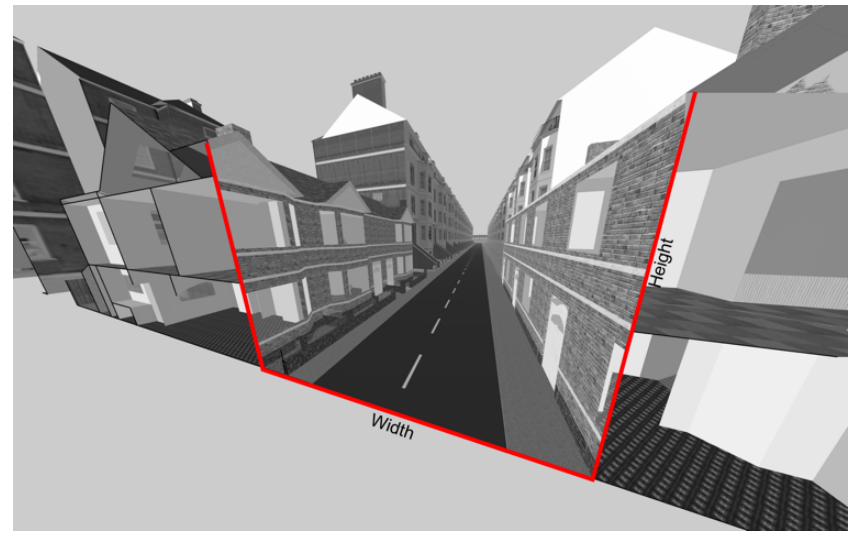

Figure 10: Street Enclosure Diagram. We define street enclosure as the ratio between avg.height/avg.width.

The results in Table 1 shows the mean squared error and $R^{2}$ for all four models when applied to a spatially random test-set. The model with 32 components achieves $60 \%$ accuracy, while the model with 4 components achieve $50 \%$ accuracy. The results show that we can achieve a good accuracy with minimal number of components in the model. However further prediction experiments are needed to confirm its usefulness for other geographical tasks.

\section{Table 1: Street Enclosure Results}

\begin{tabular}{r|c|c} 
& $R^{2}$ & $M S E$ \\
\hline 4 components (Vis) & $49.51 \%$ & 0.53 \\
\hline 8 components (Vis) & $55.76 \%$ & 0.47 \\
\hline 16 components (Vis) & $57.31 \%$ & 0.45 \\
\hline 32 components (Vis) & $59.7 \%$ & 0.43 \\
\hline & \multicolumn{2}{|c}{}
\end{tabular}

\section{2 street network}

4.2.1 Visualisation experiments. For the street network, the trained autoencoder learned a mapping from the space of street network images ( $256 \times 256 \times 1$ or 65,536 dimensions) to a lower dimensional latent space (640 dimensions) which are then further summarised into a set of linearly uncorrelated variables by applying $\left(P C A_{l i n}\right)$. By plotting out the street network images with the lowest to highest values of each component we can start to interpret the learn latent space. In figure 11, we show the first five. These plots all relate to density of streets in different spatialised regions. The first $p c a$ encodes general density, while pca 2-5 encode spatialised densities (left-right, top-bottom, center-periphery, diagonals) respectively.

To make it easier to interpret each pca we create a mean vector $\hat{v}$, where we keep all values in $\hat{v}$ constant and vary only the pca before passing it to the generator to create a synthetic image. In figure 12, we show a subset of the different visual response encoded by the pca values. We show that the first 10 pca encode regions of spatialised density, pca 11-50 encode global structure of the network (coarse grain detail), and pca 50-640 encode local structure of the network (finer grain detail).

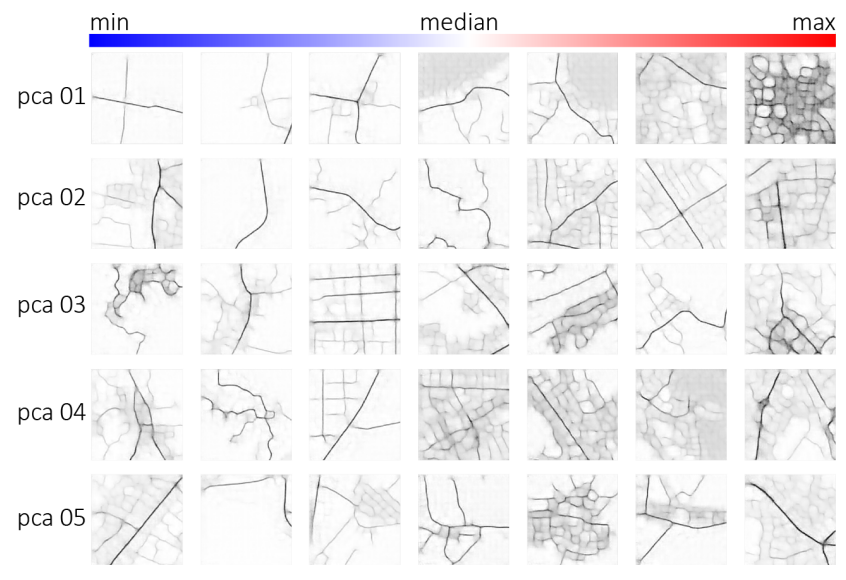

Figure 11: Example street network images for the first five principal components.

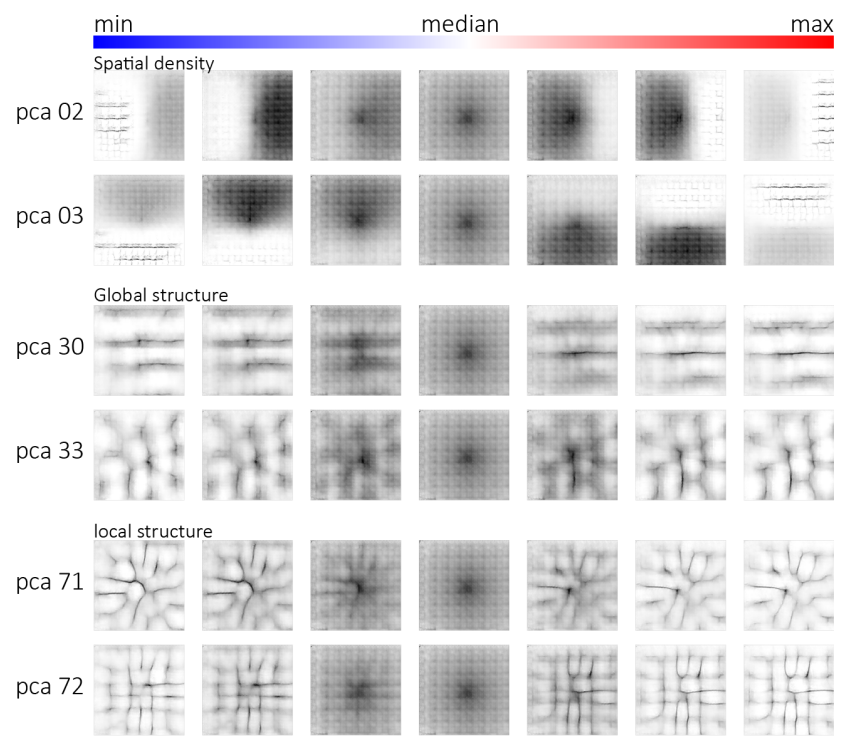

Figure 12: Visual response perturbation of an average Street Network image. By perturbing the visual response of an average image, we are able to show meaning from the perturbed component. The first sets of components seems to be related to spatialised density. While the second and third sets of components seems to be related to global and local structure of the street network.

By mapping the values of the principal components we can further test spatial patterns that they might encode. With just the first principal component of the latent space we are able to differentiate street network densities across the city of London. Figure 13 shows central London has higher street density than outer London.

4.2.2 Prediction experiment. Lastly we test the ability of these encoding to capture network features by using them to predict network statistics such as: average circuity, intersection density 


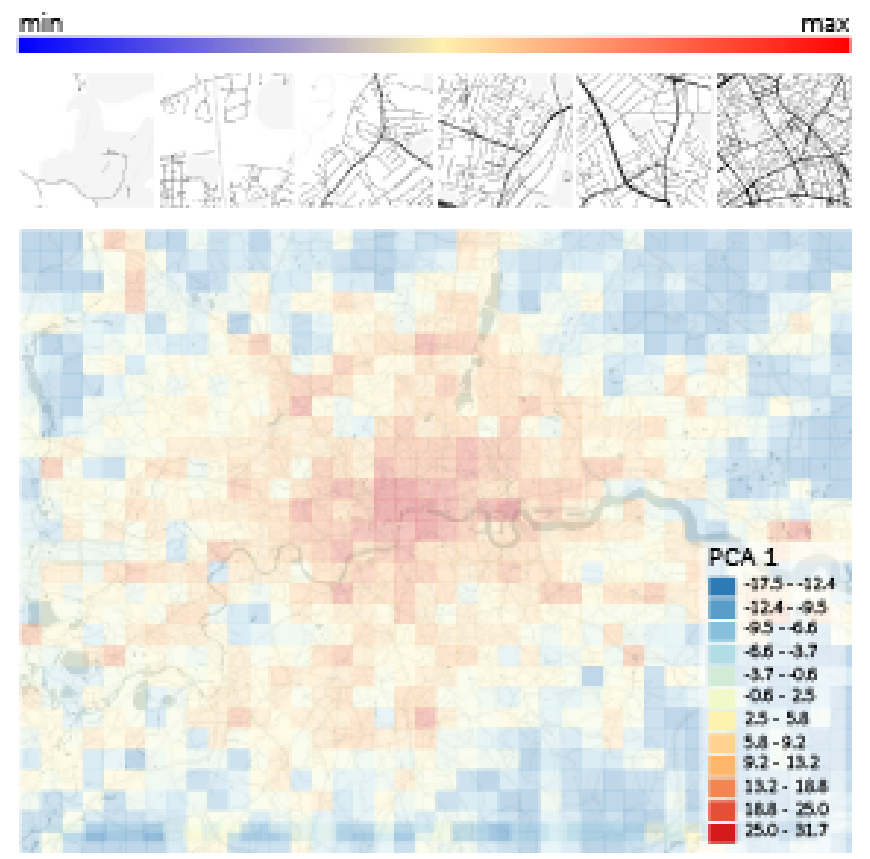

Figure 13: Values of the first principal component across London. The first component of the street network images can be interpreted as a measure of street network density.

and edge density. We define average circuity as the sum of all edge lengths by the sum of the great-circle distances between nodes incident to each edge, intersection density as the number of nodes in each grid divided by the area of the grid, and edge density as the sum of edge lengths divided by the area of the grid. For our model we train similarly a multi-layer perceptron, as specified in the previous section.

The results in Table 2 show the mean squared error and $R^{2}$ for street intersection density using different number of pca components. With just a few components we are able to achieve an accuracy of $77 \%$ on spatial features of the graph (edge density and intersection density), however the model fails to capture more complex network features such as circuity.

Table 2: Intersection density results

\begin{tabular}{r|c|c} 
& $R^{2}$ & $M S E$ \\
\hline 4 components (Vis) & $76.59 \%$ & 0.23 \\
\hline 8 components (Vis) & $77.28 \%$ & 0.22 \\
\hline 16 components (Vis) & $75.54 \%$ & 0.26 \\
\hline 32 components (Vis) & $71.15 \%$ & 0.31 \\
\hline & \multicolumn{2}{|l}{}
\end{tabular}

\section{DISCUSSION AND CONCLUSION}

We have presented a simple but novel unsupervised approach to extract and interrogate visual latent responses from urban images. This research sits in contrast to previous works which focused on supervised learning $[9,18,23,27]$ and unsupervised learning for reconstruction $[14,31]$. Through visualisation, both geographically and generative, and prediction experiments we were able to retrieve meaning from these latent responses.

In the case of the street level images, by visualising the extremes of the latent variable and perturbing it, we were able to discover meaning from the data such as the visual richness and urban density of a street. We also find that the latent variables are able to predict a generic urban characteristic such as street enclosure. However to validate the method, future works is needed on a) creating higher quality reconstructions using more advance generative methods such as $V A E$ or $G A N$ b) developing quantitative methods to systematically explain these visual latent responses and c) conducting research on multi-task and multi-modal learning for different geographical tasks.

In the case of the street networks, although the model is able to predict road network density, it fails to capture more complex street network features, we believe this is because the self-organized pattern of street networks is the result of both geometrical order/disorder as well as local rules of optimality. Through rasterising the street networks, the explicit topological data of the graph is lost, and the model is not able to recover this from the image alone. Future work can explore ways to incorporate topological properties of the networks in the model. Recent advances in graph neural networks provide promising directions that would allow both topological and geometric properties to be incorporated into the model, this would allow a richer representation of the street network as both local connectivity structure and their spatial embedding would be preserved.

An immediate implication of the study, is that by discovering general knowledge from these urban images, we can use this information for other down-stream geographical tasks. For example in using street-level images to predict land classification. Conversely, this can reduce compute time and data collection costs significantly. More importantly though, the knowledge discovery process of using a combination of visualisation and inference, can represent an exploratory approach for learning more about these complex nonlinear methods such as neural networks and higher dimensional datasets such as images.

To conclude, this research contributes to recent efforts in linking the disciplines of geography and machine learning. On the one hand, we find the visual latent responses as learnt from street level and street network images have interpretable meanings. On the other hand, we also demonstrate how geographical datasets and visualisations techniques can be used to enrich our understanding of machine learning methods.

\section{REFERENCES}

[1] Martín Abadi, Ashish Agarwal, Paul Barham, Eugene Brevdo, Zhifeng Chen, Craig Citro, Greg S. Corrado, Andy Davis, Jeffrey Dean, Matthieu Devin, Sanjay Ghemawat, Ian Goodfellow, Andrew Harp, Geoffrey Irving, Michael Isard, Yangqing Jia, Rafal Jozefowicz, Lukasz Kaiser, Manjunath Kudlur, Josh Levenberg, Dan Mané, Rajat Monga, Sherry Moore, Derek Murray, Chris Olah, Mike Schuster, Jonathon Shlens, Benoit Steiner, Ilya Sutskever, Kunal Talwar, Paul Tucker, Vincent Vanhoucke, Vijay Vasudevan, Fernanda Viégas, Oriol Vinyals, Pete Warden, Martin Wattenberg, Martin Wicke, Yuan Yu, and Xiaoqiang Zheng. 2015. TensorFlow: Large-Scale Machine Learning on Heterogeneous Systems. https://www.tensorflow.org/ Software available from tensorflow.org.

[2] Michael Batty. 2005. Cities and complexity: understanding cities through cellular automata, agent-based models and fractals. 
[3] Yoshua Bengio, Pascal Lamblin, Dan Popovici, and Hugo Larochelle. 2007. Greedy Layer-Wise Training of Deep Networks. In Advances in Neural Information Processing Systems 19, B. Schölkopf, J. C. Platt, and T. Hoffman (Eds.). MIT Press, 153-160. http://papers.nips.cc/paper/ 3048-greedy-layer-wise-training-of-deep-networks.pdf

[4] Geoff Boeing. 2017. OSMnx: New methods for acquiring, constructing, analyzing, and visualizing complex street networks. Computers, Environment and Urban Systems 65 (2017), 126-139.

[5] Geoff Boeing. 2018. Measuring the Complexity of Urban Form and Design. October (2018), 1-22.

[6] Geoff Boeing. 2018. A multi-scale analysis of 27,000 urban street networks: Every US city, town, urbanized area, and Zillow neighborhood. Environment and Planning B: Urban Analytics and City Science (2018), 2399808318784595

[7] François Chollet. 2015. keras. https://github.com/fchollet/keras.

[8] A Dubey, N Naik, D Parikh, R Raskar, and C Hidalgo. 2016. Deep Learning the City : Quantifying Urban Perception At A Global Scale. European Conference on Computer Vision (ECCV) (2016).

[9] T Gebru, J Krause, Y Wang, D Chen, J Deng, E Aiden, and F Li. 2017. Using deep learning and Google Street View to estimate the demographic makeup of neighbourhoods across the United States. PNAS (2017).

[10] Google. 2018. https://www.maps.google.com/.

[11] Zhu E. Yin J. Guo X., Liu X. 2017. Deep Clustering with Convolutional Autoencoders. Neural Information Processing. ICONIP 2017. Lecture Notes in Computer Science (2017).

[12] Stefan Hartmann, Reinhard Klein, Raoul Wessel, and Reinhard Klein. 2017. StreetGAN : Towards Road Network Synthesis with Generative Adversarial Networks. (2017).

[13] Bill Hillier. 2007. Space is the machine: a configurational theory of architecture Space Syntax.

[14] Phillip Isola, Jun-Yan Zhu, Tinghui Zhou, and Alexei A. Efros. 2018. Imageto-Image Translation with Conditional Adversarial Networks. (2018). https: //arxiv.org/abs/1611.07004

[15] Kira Kempinska and Murcio Roberto. 2019. Modelling urban networks using Variational Autoencoders. arXiv preprint (2019). arXiv:arXiv:1905.06465v1

[16] Diederik P. Kingma and Jimmy Ba. 2014. Adam: A Method for Stochastic Optimization. arXiv:cs.LG/1412.6980

[17] Konstantin Klemmer, Adriano Soares Koshiyama, and Sebastian Flennerhag. 2019 Augmenting correlation structures in spatial data using deep generative models. ArXiv abs/1905.09796 (2019).
[18] Stephen Law, Brooks Paige, and Chris Russell. 2018. Take a look around:Using Street View and Satellite Images to Estimate House Prices. arXiv preprint (2018) https://arxiv.org/abs/1807.07155

[19] Rémi Louf and Marc Barthelemy. 2014. A typology of street patterns. Fournal of The Royal Society Interface 11, 101 (2014), 20140924.

[20] Jonathan Masci, Ueli Meier, Dan Cireşan, and Jürgen Schmidhuber. 2011. Stacked Convolutional Auto-encoders for Hierarchical Feature Extraction. In Proceedings of the 21th International Conference on Artificial Neural Networks - Volume Part I (ICANN'11). Springer-Verlag, Berlin, Heidelberg, 52-59. http://dl.acm.org/ citation.cfm?id=2029556.2029563

[21] Harvey J. Miller and Jiawei Han. 2001. Geographic Data Mining and Knowledge Discovery. Taylor \& Francis, Inc., Bristol, PA, USA

[22] Vahid Moosavi. 2017. Urban morphology meets deep learning : Exploring urban forms in one million cities, town and villages across the planet. arXiv preprint (2017), 1-10. arXiv:arXiv:1709.02939v2

[23] N. Naik, J. Philipoom, R. Raskar, and C.A. Hidalgo. 2014. StreetScore - Predicting the Perceived Safety of One Million Streetscapes. In CVPR Workshop on Web-scale Vision and Social Media.

[24] OpenStreetMap contributors. 2019. Planet dump retrieved from https://planet.osm.org . https://www.openstreetmap.org.

[25] M. Reichstein, G. Camps-Valls, B. Stevens, M. Jung, J. Denzler, N. Carvalhais, and Prabhat. 2018. Deep learning and process understanding for data-driven Earth system science. Nature (2018). http://www.nature.com/articles/s41586-019-0912-1.

[26] German Ros, Laura Sellart, Joanna Materzynska, David Vazquez, and Antonio M. LopezâĂăăĂą. 2016. The SYNTHIA Dataset: A Large Collection of Synthetic Images for Semantic Segmentation of Urban Scenes. (2016). refbase.cvc.uab.es/ files/RSM2016.pdf

[27] C Seresinhe, T Preis, and S Moat. 2017. Using deep learning to quantify the beauty of oudoor places. Royal Society Open Science (2017)

[28] Karen Simonyan and Andrew Zisserman. 2014. Very deep convolutional networks for large-scale image recognition. arXiv preprint arXiv:1409.1556 (2014)

[29] Emanuele Strano, Vincenzo Nicosia, Vito Latora, Sergio Porta, and Marc Barthélemy. 2012. Elementary processes governing the evolution of road networks. Scientific reports 2 (2012), 296

[30] Ordnance Survey. 2017. https://www.ordnancesurvey.co.uk/opendatadownload/ products.html.

[31] Ting-Chun Wang, Ming-Yu Liu, Jun-Yan Zhu, Nikolai Yakovenko, Andrew Tao, Jan Kautz, and Bryan Catanzaro. 2018. Video-to-Video Synthesis. In NeurIPS. 